This is a self-archived version of an original article. This version may differ from the original in pagination and typographic details.

Author(s): Heimbürger, Anneli; Kiyoki, Yasushi

Title: On Temporal Aspects in Cross-Cultural e-Collaboration Between Finland and Japan Research Teams

Year: 2018

Version: Published version

Copyright: ㄷ 2018 IGI Global.

Rights: In Copyright

Rights url: http://rightsstatements.org/page/InC/1.0/?language=en

Please cite the original version:

Heimbürger, A., \& Kiyoki, Y. (2018). On Temporal Aspects in Cross-Cultural e-Collaboration Between Finland and Japan Research Teams. International Journal of E-Collaboration, 14(2), 3754. https://doi.org/10.4018/ijec.2018040103 


\title{
On Temporal Aspects in Cross- Cultural e-Collaboration Between Finland and Japan Research Teams
}

\author{
Anneli Heimbürger, University of Jyväskylä, Jyväskylä, Finland \\ Yasushi Kiyoki, Keio University (SFC), Fujisawa, Japan
}

\begin{abstract}
Time is an essential dimension in cross-cultural e-collaboration among research project teams. Understanding temporal aspects and project dynamics in cross-cultural research e-collaboration and related processes can improve team members' skills in cross-cultural communication and increase their cultural competence. The present case cultures are Finnish and Japanese, and the case universities are the University of Jyväskylä (Finland) and Keio University (Japan). Three issues are addressed in this article. First, cultural dimensions and time models in the cross-cultural e-collaboration context are discussed. Second, temporal aspects related to e-collaboration activities are introduced. Third, formal, ontological approaches for identifying and describing temporal entities in cross-cultural e-collaboration are presented and examples of applications are given. The objectives of this article are (1) to deepen the knowledge and understanding of temporal aspects (informal and formal) in a cross-cultural e-collaboration environment (CCeCE) and (2) to create know-how for designing CCeCE-like systems.
\end{abstract}

\section{KEYWORDS}

Cultural Dimensions, Finland, Japan, Multidisciplinary, OWL-Time, Temporal Entities, Temporal Regions, Time Models, Time Ontology

\section{INTRODUCTION}

Because of globalization, Asian and Western cultures meet each other increasingly in connection with business, research, environmental protection, emergency situations, higher education and medical care. Cross-cultural actions are carried out in the virtual world via e-mails, web meetings and collaborative virtual work spaces, as well as in the physical world with face-to-face meetings. In situations like these, people may experience cultural differences related to business, research and social etiquette, language structure, thought structure, attitudes toward time, personal space, facesaving, communication style, acceptance and use of silence. What is culture? According to Wang (2009), culture is embodied in how people interact with other individuals and with their environment; culture is a way of life formed under specific historical, natural and social conditions.

Cultural sensitivity has become an important dimension for success in today's international research arena. Despite the globalization trend, research project managers, project teams and 
team members may find themselves uncertain in situations due to culture-dependent differences in communication protocol, language and value systems (Berry et al., 2011; Shaules, 2015). Consequently, people involved in cross-cultural project transactions are usually advised to be aware of their colleagues' cultural backgrounds. Cultural competence might help project teams achieve project goals and support the teams to promote a creative and motivated atmosphere throughout the whole collaboration.

Today, international joint research projects are often carried out in e-Collaboration environments defined as "collaboration, which is conducted without face-to-face interaction among members of virtual teams engaged in a common research task using information and communication technologies (ICT)" (Adam, 2006; Bettoni et al., 2015; Johnson and Keane, 2017). E-Collaboration is increasing not only because of its economic and environmental benefits but also due to its flexibility for establishing dynamically new cross-organizational and -cultural innovative teams (Watanabe, 2017). In virtual collaborative spaces, geographic borders and time zones are easy to cross. In addition, such spaces should support members' joint activities. To model, design and realize these spaces, an understanding of the processes to be carried out by the e-Collaboration community is necessary, as well as an understanding of the related cultures, contexts and project knowledge. In e-Collaboration, the importance of cultural awareness and sensitivity are emphasized because of the lack of face-toface communication.

In joint research projects between the University Y (Japan) and the University X (Finland), the ultimate objective is to model, design and implement a prototype of a cross-cultural e-Collaboration environment $(\mathrm{CCeCE})$ that will support cross-cultural research teams in communication, in joint activities and in knowledge creation, sharing and management. The case cultures are Finnish and Japanese. For realizing a CCeCE, different kinds of approaches, models, methods and technologies were tested. The whole system includes several aspects (team manager and team members, processes and technology) as illustrated in Figure 1. The focus of this article is on temporal aspects related to cross-cultural e-Collaboration, and especially do the competence of cross-cultural temporal aspects between the team members improve smooth e-Collaboration. The study results indicate that indepth understanding and knowledge about temporality among team leaders and team members in cross-cultural e-Collaboration context promote efficient and successful team working and projects' implementations according to give schedules.

The article is organized as follows. First, cultural models and their implications for crosscultural e-Collaboration are introduced. Secondly, time models in cultural contexts and temporality in e-Collaboration are discussed. As a formal approach, the authors define temporal entities and describe OWL-Time ontology, temporal aggregates (an extension of OWL-Time) and the temporal

\section{Figure 1. Cross-cultural e-Collaboration environment}

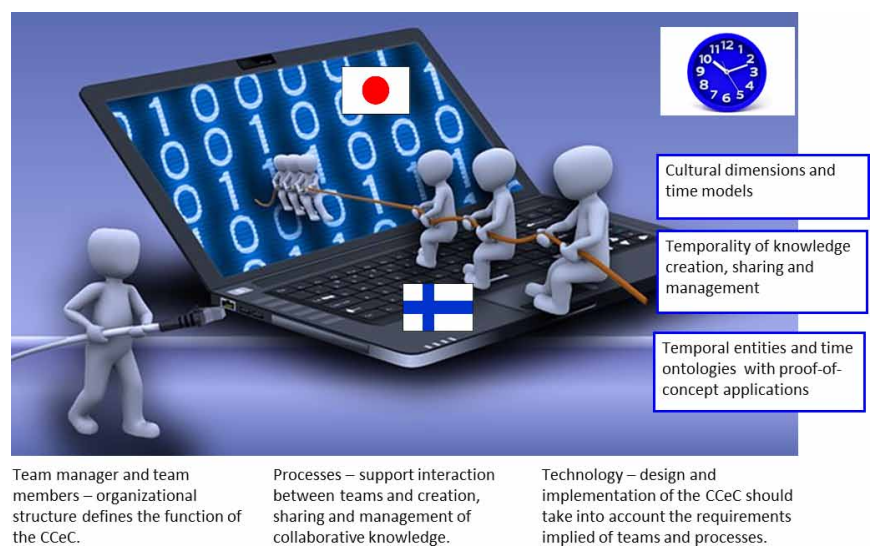


component of the Basic Formal Ontology (BFO). They also present proof-of-concept examples of the approaches in cross-cultural e-Collaboration between Finnish and Japanese teams.

Approaches of the first part of the paper (cultural dimensions and time models in cultural context) are more informal. The second part of the paper (temporality in e-collaboration and temporal entities) presents semi-formal approaches and finally, the third part of the paper (time ontologies) presents formal approaches to temporal aspects in cross-cultural e-Collaboration. With this structure, the article aims to indicate on the one hand the diversity and multidisciplinary of temporal aspects' research and on the other hand aims to form step by step ensemble of the topic at issue - starting from informal, proceeding to semi-formal and ending up to formal approaches. In addition to literature review (related work), the article has elements of case study and design science research.

\section{CULTURAL DIMENSIONS}

By integrating the three main, also most cited, cultural models: Hall's (1990) model, Hofstede's (2010) model and Lewis's (2010) model, Parrish and Linder-VanBerschot (2010) outlined eight cultural dimensions. The authors divide these dimensions into three main categories that are most likely to affect cross-cultural e-Collaboration and team work processes:

1. Social relationships:
a. Equality and authority;
b. Individualism and collectivism;
c. Nurturing and challenge;

2. Epistemological beliefs:
a. Stability-seeking and uncertainty acceptance;
b. Logic argumentation and rationality;
c. Causality and complex systems;

3. Temporal perceptions:
a. Clock and event time;
b. Linear and cyclical time.

From this research point of view, Hofstede's and Lewis's models are the most interesting. Lewis's model focuses on multi-cultural communication. He divided the world's cultures into three rough categories: (1) linear-active cultures, (2) multi-active cultures and (3) reactive cultures. Linear-active cultures plan, schedule, organize, pursue action chains and do one thing at a time. Members of multiactive cultures are lively, loquacious people who do many things at once, planning their priorities not according to a time schedule but in accordance with the relative importance of each appointment. Reactive cultures prioritize courtesy and respect, listening quietly and calmly to their interlocutors and reacting carefully to the other side's proposals. According to Lewis's model, Finland is close to a linear-active culture and Japan to a reactive culture.

Hofstede's approach proposes a set of cultural dimensions along which dominant value systems can be ordered. The framework consists of six dimensions or indexes (Hofstede, 2010):

1. Power distance is the extent to which the less powerful members of organizations and institutions accept and expect that power is distributed unequally;

2. Individualism/collectivism explores the degree to which people in a society are integrated into groups;

3. Uncertainty avoidance is a society's tolerance for ambiguity, in which people embrace or avert an event of something unexpected, unknown or different from the status quo;

4. In the masculinity/femininity dimension, masculinity is defined as a preference in society for achievement, heroism, assertiveness and material rewards for success. The counterpart represents a preference for cooperation, modesty, caring for the weak and quality of life; 
5. Long-term orientation/short-term orientation associates the connection of the past with current and future actions/challenges. A lower degree of this index (short-term) indicates that traditions are honored and kept, while steadfastness is valued. Societies with a high degree in this index (long-term) view adaptation and circumstantial, pragmatic problem-solving as a necessity;

6. Indulgence/restraint is a measure of happiness-whether or not simple joys are fulfilled. Indulgence is defined as a society that allows relatively free gratification of basic and natural human desires related to enjoying life and having fun. The counterpart is defined as a society that controls gratification of needs and regulates it with strict social norms.

As an example, the cultural index scores (scale 0-100) for Finland and Japan are shown in Figure 2. The survey and the tool for calculating cultural indexes (https://www.hofstede-insights.com/countrycomparison/) are extensively described in Hofstede (2019). The figures should not be taken literally.

Although cultural models are generalizations and individuals may vary from their society's descriptors, Hofstede's and Lewis's approaches provide interesting frameworks and approaches for cultural understanding. Different value systems affect human thinking, learning, feeling and acting, and the behavior of teams and organizations, as well as the temporal aspects of cross-cultural e-Collaboration. In Table 1, the authors summarize possible implications of cross-cultural differences for e-Collaboration, highlighting temporal issues, according to Hofstede's dimensions.

Figure 2. The scores for Finland (first column) and Japan (second column) according to Hofstede's cultural indexes

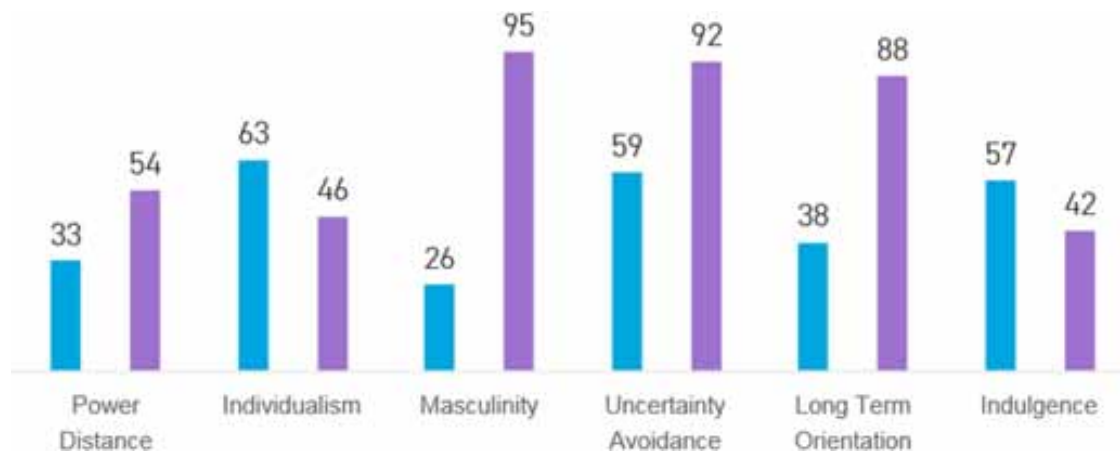

Table 1. Possible implications of cross-cultural differences in e-Collaboration

\begin{tabular}{|l|l|}
\hline \multicolumn{1}{|c|}{ Dimension } & \multicolumn{1}{c|}{ Possible Implication } \\
\hline $\begin{array}{l}\text { Individualism/ } \\
\text { collectivism }\end{array}$ & $\begin{array}{l}\text { Team members from a collectivistic society are likely to spend more time on long-term goals. Conversely, team members } \\
\text { from individualistic societies are more likely to focus on short-term goals. }\end{array}$ \\
\hline Power distance & $\begin{array}{l}\text { Team members from low power-distance cultures may be frustrated by the need for team members from high power- } \\
\text { distance cultures to seek consensus and approval from higher authority. The process can be time-consuming. }\end{array}$ \\
\hline $\begin{array}{l}\text { Masculinity/ } \\
\text { femininity }\end{array}$ & $\begin{array}{l}\text { Team members from masculine cultures are more likely to be competitive and ready for quick solutions, and those from } \\
\text { feminine cultures to be empathic and seek compromises in the long run. }\end{array}$ \\
\hline $\begin{array}{l}\text { Uncertainty } \\
\text { avoidance }\end{array}$ & $\begin{array}{l}\text { Team members from high risk-avoidance cultures are likely to view those from low risk-avoidance cultures as unfocused. } \\
\text { Those from low risk-avoidance cultures are likely to view team members from high risk-avoidance cultures as rigid. This } \\
\text { may affect the progress of the project because decision making takes time. }\end{array}$ \\
\hline $\begin{array}{l}\text { Long-term/short- } \\
\text { term orientation }\end{array}$ & $\begin{array}{l}\text { Team members in long-term-oriented cultures are accustomed to working toward building strong positions in their } \\
\text { collaboration and do not expect immediate results. In short-term-oriented cultures, the results of the past month, quarter or } \\
\text { year are a major concern. }\end{array}$ \\
\hline Indulgence/ restraint & $\begin{array}{l}\text { Team members in indulgence-oriented cultures may be more flexible in scheduling e-Collaboration activities than team } \\
\text { members in restraint-oriented cultures. }\end{array}$ \\
\hline
\end{tabular}




\section{TIME MODELS IN CULTURAL CONTEXTS}

Time is seen in a different way by Asian and Western cultures, and even within these groupings, temporal culture differs from country to country. In addition, the temporal identities of different organizations and teams in organizations may vary. In the cultural context, according to Lewis (2010) four time models exist: linear time, cyclic time, multi-active time and back to the future. From the case cultures' point of view, the linear and cyclic time models are the most essential.

In the linear time model (Figure 3a), the past time is over, and the present time can be seized, divided up and made to work for the immediate future. One task is carried out at a time. For example, Finns are essentially linear-active, time-dominated and monochronic. They prefer to do one thing at a time, concentrate on it and do it on a schedule.

Cyclic time sees time as circular, not necessarily leading toward something but repeating itself in a cycle of events. Each day, the sun rises and sets, the seasons follow one another, people grow old and die, but their children reconstitute the process. In many Asian countries, time has traditionally been considered cyclic. For example, the Japanese traditional temporal culture can be represented by the Makimono model of time (Figure 3b) (De Mente, 2004; Ghauri and Usunier, 2003). Makimono is a type of Japanese hand scroll that unrolls horizontally, or laterally on a flat surface. In Makimono time, the future flows into the present, just as the past does. The present is a period that links the region of the past to the world of the future. Today, the linear time model has also been integrated into Japanese society. At present, Japan uses the Gregorian calendar, together with year designations stating the year of the reign of the current emperor.

Linear time is believed to be the closest depiction of Finnish experience of time. However, for many centuries Japan used a cyclical view of time that supports the way nature behaves. Western linear-type action chains and Asian reflection are compared in Figure 4 (Lewis, 2010). The Western

Figure 3. Linear time model (a) and cyclic time model (b)

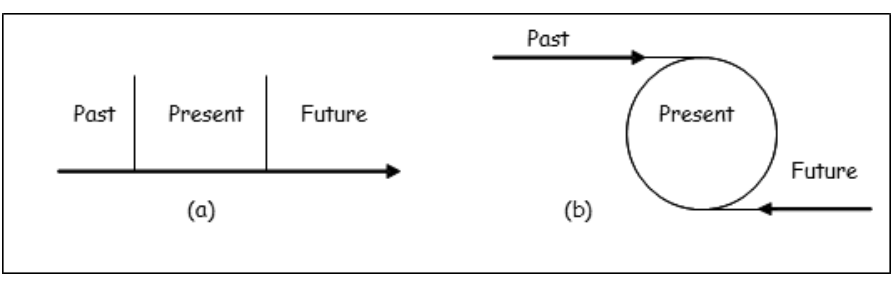

Figure 4. Western action chains versus Asian reflection according to Lewis (2010)

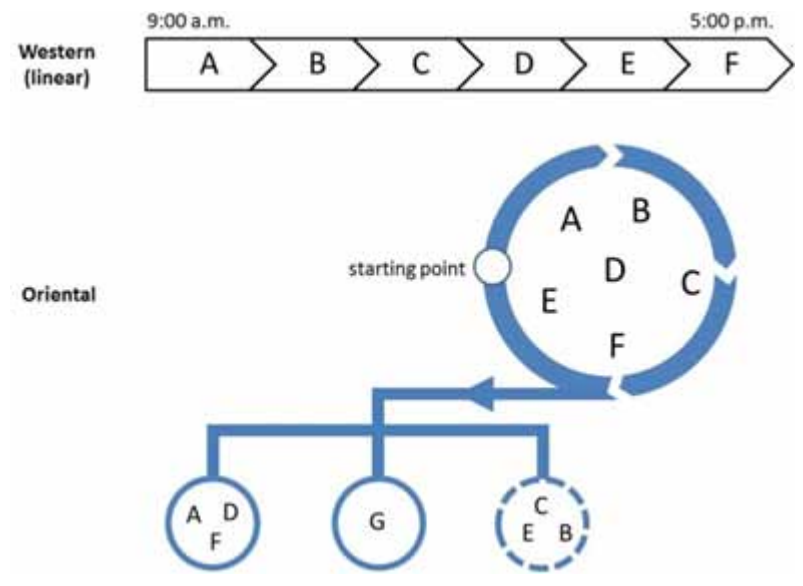


model contains tasks A-F to be sequentially completed during the day. In Asian reflection, instead of tackling problems immediately in sequence, circling round them for some time is preferred. After a suitable period of reflection, A, D and F may seem worth pursuing. B, C and E may be quietly dropped. Contemplation of the whole scene has indicated, however, that task $\mathrm{G}$ might be the most significant of all.

To summarize the main difference between Finland's and Japan's sense of time, in Finland time orientation emphasizes objectivity, absoluteness and fixation of time. In Japan, the traditional cultural time orientation conceives of time as subjective, relative and flexible. The understanding of a cultural concept of time is an issue for successful cross-cultural communication and crosscultural e-Collaboration.

\section{TEMPORALITY IN E-COLLABORATION}

A research project in the e-Collaboration context can be defined as a combination of human and non-human resources pulled together into a temporary organization to achieve a specified purpose (Bosch-Sijtsema and Henriksson, 2014; Desouza and Evaristo, 2004). The project team manager is the chief executive of this temporary organization. According to the project management typology introduced by Evaristo and van Fenema (1999), the authors illustrate in Figure 5 distributed e-collaboration. It can be divided into two main categories: single and multiple e-collaborations. Furthermore, multiple e-collaborations can be divided into multiple traditional e-collaborations, multiple co-located e-collaborations, multiple distributed e-collaborations with discrete locations and multiple distributed e-Collaborations with shared locations.

An e-collaboration environment usually consists of four main layers: (1) a knowledge creation, sharing and management layer, (2) a communication layer, (3) a layer for operations in e-Collaboration and (4) a performance layer. The performance layer implements tools for processing knowledge across the whole e-collaboration environment according to the working strategy and culture of the participating teams. The layer for operations in e-Collaboration provides tools and support for general management issues. The communication layer implements tools for keeping team members informed about what other members are doing and thus, increases transparency and information transfer in e-collaboration. The knowledge creation, sharing and management layer constitutes the repository for e-collaboration deliverables with knowledge classification and lifecycle management.

The tasks of the research project in e-collaboration reflect the knowledge in the project. Three types of task interactions can be observed: independent, dependent and coupled (Chen et al., 2003). Tasks are independent when no information exchange between them is required. These tasks can

Figure 5. E-collaboration typology according to Evaristo and van Fenema (1999)

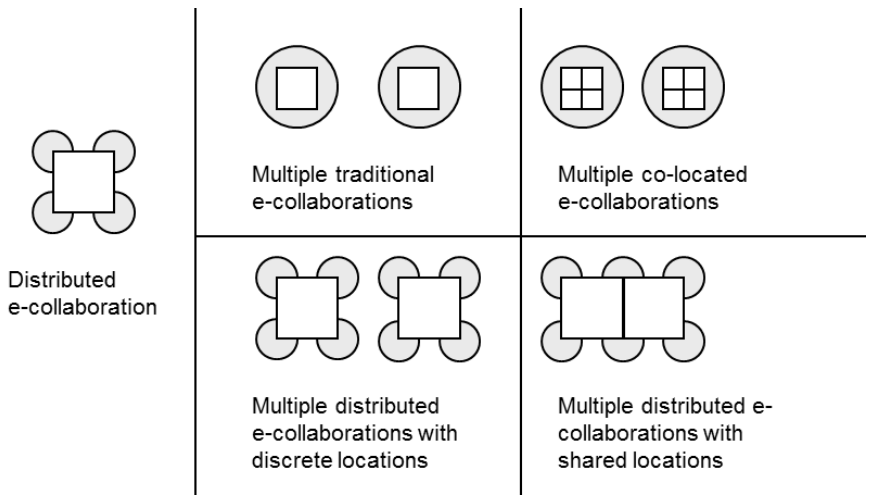


be executed simultaneously. Dependent tasks transfer information sequentially and typically are performed in series. Coupled tasks are interdependent and need multiple iterations to complete. The tasks in an e-collaborative project are positioned in the project schedule. They can have several temporal characteristics (Shipp and Fried, 2014) as follows.

Duration is the amount of time spent on completing a task. The temporal location of activities and tasks can be situated at particular points over the continuum of time, when they take place. A sequence means the order in which tasks take place. A deadline is a fixed time by when the work is to be completed. A cycle means periodic regularity in which work is completed repeatedly. A time interval between repetitions can be constant or a function of time. A rhythm describes an alternation in the intensity of being busy.

Knowledge creation, sharing and management statuses and timed status transitions are examples of monitoring functions of e-Collaboration progress from the temporal point of view. A knowledge status describes the stage reached in the knowledge's life cycle and is used to control the releasing process by limiting user access privileges in each status. Knowledge statuses can be defined as new, in work, for approval, approved, released and obsolete. A timed status transition is a mechanism for defining a valid time interval $[\mathrm{t}$ (yyyy/mm/dd/hhmmss) 1 , $\mathrm{t}(\mathrm{yyyy} / \mathrm{mm} / \mathrm{dd} / \mathrm{hhmmss}) 2]$. For example, a piece of knowledge can be in the "in work" status two weeks starting from $\mathrm{t} 1=2019 / 09 / 01 / 080000$ and ending at $\mathrm{t} 2=2019 / 09 / 15 / 160000$, and then the knowledge is automatically moved to the "for approval" status. Granularity levels of time related to knowledge (document) statuses are summarized in Table 2. Granularity levels of temporal rules related to the document lifecycle are shown in Table 3.

Table 2. Granularity levels of time related to document statuses

\begin{tabular}{|l|l|}
\hline \multicolumn{1}{|c|}{ Level } & \multicolumn{1}{c|}{ Definition } \\
\hline New & A document is created in a given time instant. \\
\hline In work & Work is under construction. \\
\hline For approval & Authorities evaluate the document(s). \\
\hline Released & A document has been approved. \\
\hline Obsolete & $\begin{array}{l}\text { A document has become outdated. In some cases, a new version is created, and its } \\
\text { critical time intervals for validity defined. }\end{array}$ \\
\hline
\end{tabular}

Table 3. Granularity levels of temporal rules related to the document lifecycle

\begin{tabular}{|l|l|}
\hline \multicolumn{1}{|c|}{ Level/Rule } & \multicolumn{1}{c|}{ Definition } \\
\hline Ready before & A document must be ready before a given time instant or time interval. \\
\hline Ready right before & A document must be ready just before a given time instant or time interval. \\
\hline Ready after & A document must be ready after a given time instant or time interval. \\
\hline Ready right after & A document must be ready just after a given time instant or time interval. \\
\hline Ready equal & A document must be ready at a given time instant. \\
\hline Overlap & Two documents or document groups share the same status during a given time interval. \\
\hline Within & $\begin{array}{l}\text { Documents with a certain status completely within a given interval are chosen. In } \\
\text { “overlap," the status of a document may also exceed the given interval. }\end{array}$ \\
\hline Start & Documents whose lifecycles start at a given time instant. \\
\hline Finish & Documents whose lifecycles end at a given time instant. \\
\hline
\end{tabular}


An example of the "ready right before" rule is if the temporal rule in R-before is 20.9.2019 to 22.9.2019, then the resulting set of documents are those that end on 20.9.2019, and if R-after is chosen, the resulting set of documents are those that start on 22.9.2019. The difference between the Before/After temporal rules and R-Before/R-After is that in R-Before/R-After documents are stuck in the edge of the given interval. The Before-rule also selects documents that ended long before the given time interval. The terminology is consistent with Allen's relations between two time intervals (Allen, 1983). Allen's interval algebra defines thirteen possible relations between time intervals as shown in Figure 6.

In cross-cultural e-Collaboration, Allen's relations can be illustrated by two research teams' joint activities (Figure 7).

At a general level, different kinds of time contexts related to cross-cultural e-Collaboration a team manager may face exist (Figure 8) (Heimbürger, 2009, 2014).

Time attitudes include cultural, organizational, group-specific and personal issues. Monochronic cultures differ from polychronic cultures in that the former encourage a highly structured, time-ordered approach to life and the latter a more flexible, indirect approach, based on personal relationships rather than scheduled commitments (Lewis, 2010). Despite globalization, many temporal habits, calendars and clocks coexist. There are many differences between calendars and clocks, such as the beginning and end of a year or academic terms. Thus, even when a team member is located in a different country, he or she may celebrate the New Year on the day the holiday is celebrated in his or her country of origin. Although e-Collaboration events may be marked on the Gregorian calendar, many social, personal and even professional events may be marked on or driven by a local calendar.

A team manager has to optimize harmonization with the local time contexts. However, he or she cannot compromise on the project's overall goals. At a fundamental level, the project schedule is simply another calendar that is specific to that project. The schedule may be implemented based on UTC (Coordinated Universal Time), a standard time common to every place in the world (Wikipedia, n.d.b). The UTC-based calendar can coexist with local calendars by adapting to them with minimum conflict, by including the events of local calendars and by working around them as additional constraints.

Figure 6. Relations between intervals according to Allen's analysis (Allen, 1983)

\begin{tabular}{|c|c|c|c|}
\hline Relation & Symbol & Inverse & Example \\
\hline$X$ before $Y$ & $<$ & $>$ & $X X X \quad Y Y Y$ \\
\hline$X$ equal $Y$ & $=$ & $=$ & $\begin{array}{l}x x x \\
y y y\end{array}$ \\
\hline$X$ meets $Y$ & M & $\mathrm{Ml}$ & XXXYYYY \\
\hline X overlaps Y & 0 & Ol & $\begin{array}{c}\mathrm{xxx} \\
\mathrm{YYY}\end{array}$ \\
\hline$X$ during $Y$ & D & DI & $\begin{array}{c}x \mathrm{xx} \\
\text { unyryy }\end{array}$ \\
\hline$X$ starts $Y$ & $S$ & SI & \begin{tabular}{|l|l|}
$x x x$ \\
yryyry
\end{tabular} \\
\hline$X$ finishes $Y$ & $\mathrm{~F}$ & $\mathrm{FI}$ & $\begin{array}{r}x \times x \\
\text { mrwry }\end{array}$ \\
\hline
\end{tabular}

Figure 7. Research team activities illustrated by Allen's relations between intervals

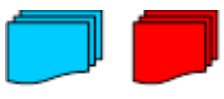

bef ore

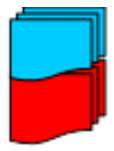

equal

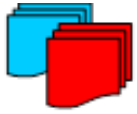

overlaps

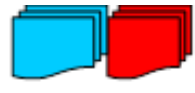

meets

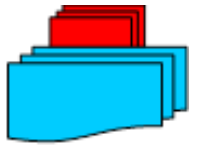

during 


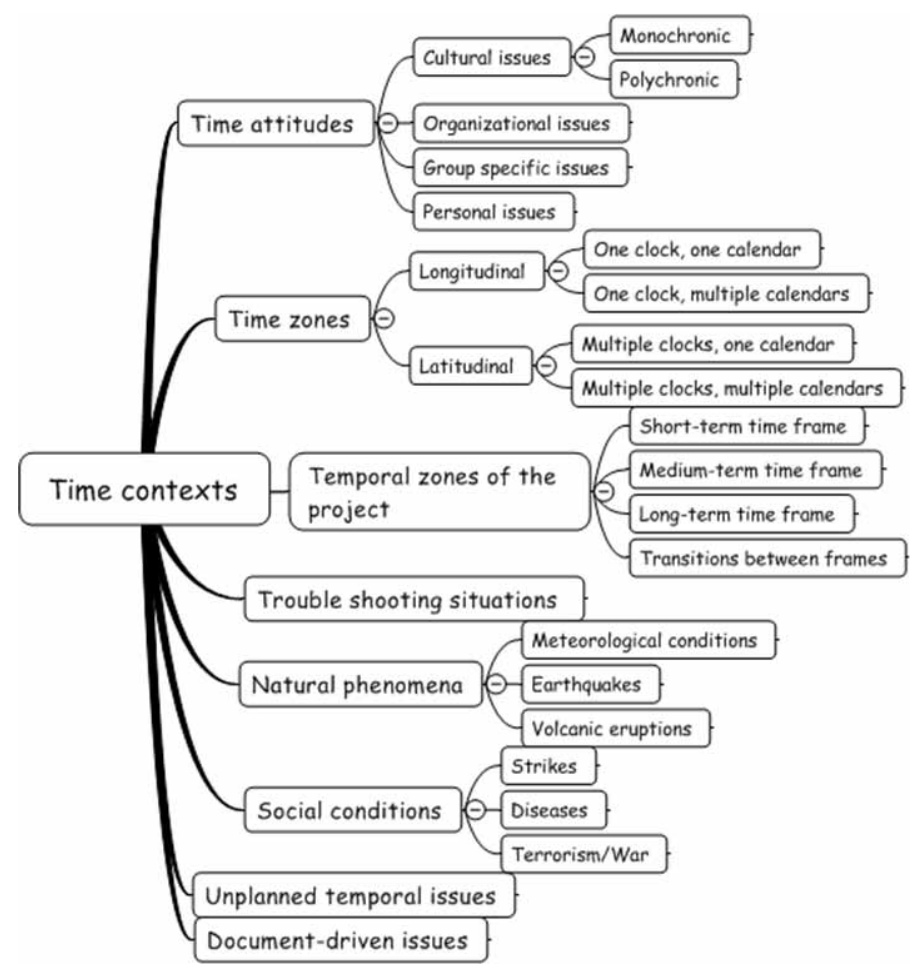

Anacona et al. (2001) introduced the concept of temporal zones in organizations. Three zones were identified: short-, medium- and long-term zones. Temporal zones applied to e-Collaboration reflect activities that share the same temporal parameters, such as pace, time horizon and cycle. In short-term time zones, acute and critical tasks are carried out, such as trouble-shooting situations in ICT. They may have effects on project scheduling. Long-term zones include planning tasks.

Natural phenomena and social conditions can be anticipated to some extent. They may have critical effect on e-Collaboration. A new invention is the example of a totally unplanned temporal issue that a team manager may have to solve, and therefore, may result in re-estimation of the realization of the whole project.

Takeuchi (2001) pointed out one of the fundamental differences between Western project managers and Asian project managers, and the difference between knowledge creation and knowledge management. Western managers emphasize the importance of explicit knowledge whereas, for instance, Japanese intellectual tradition places a strong emphasis on tacit knowledge, seeing the explicit knowledge as being just the tip of the iceberg. The distinction between explicit and tacit knowledge is the key to understanding the differences between Western and Asian approaches to knowledge. To be communicated and shared within e-Collaboration, tacit knowledge must be converted into words, drawings or numbers that everyone can understand. During the time that this kind of conversation and conversion process takes place (from tacit to explicit), project- and task-specific and more widely, team or organizational knowledge is created in cross-cultural e-Collaboration.

In the next sections, the authors explore temporal aspects of cross-cultural e-Collaboration with ontologies that provide a formal approach for this study. Proof-of-concept applications are also introduced. 


\section{TEMPORAL ENTITIES}

A temporal thing or an entity describes a point in time, event or time period at the conceptual level (Tang et al., 2010). Objects, such as people, are not temporal entities. However, objects can have a time-of-existence property that is a temporal entity, in particular, related to situations. The identification of temporal entities depends on the context. The identification approach is based on extracting the named entity that determines temporal expressions. A temporal expression is a sequence of tokens that represent an instance of a temporal entity. If the context is knowledge management in e-collaboration settings, then identifying temporal entities involves a linguistic analysis of the knowledge. If the context is a situation or a process, such as a meeting or a discussion process, then identifying temporal entities involves progressively analyzing the situation or the process. Similar to Alonso et al.'s (2007) approach, the authors identify the following three main categories of temporal expressions in this study:

1. Explicit temporal expressions: These temporal expressions directly describe entries in some timeline, such as an exact date or year. For example, the token sequences "January 2019" and "September 14, 2019, 10.00 a.m." are explicit temporal expressions and can be mapped directly to a timeline;

2. Implicit temporal expressions: Depending on the underlying time ontology and capabilities of the named entity extraction approach, even apparently imprecise temporal information, such as names of holidays or events, can be anchored in a timeline. For example, the token sequence "Ocean Day 2019 in Japan" can be mapped to the expression "July 15, 2019," or the sequence "Midsummer Day 2019 in Finland" can be mapped to "June 22, 2019." Implicit temporal expressions can also be collections of temporal entities such as "every other Wednesday every second month;"

3. Relative temporal expressions: These temporal expressions represent temporal entities that can be anchored in a timeline only in reference to another explicit or implicit, already anchored temporal expression (e.g., the start time of a meeting). For example, the expression " 3 p.m." alone cannot be anchored in any timeline. However, the expression can be anchored if the date of the meeting is known. This date then can be used as a reference for that expression, which can be mapped to a timeline. Allen's (1983) temporal calculus can be used to express relationships between relative temporal expressions.

When a system like a CCeCE are designed, formal expressions for temporalities are needed. What is the best approach? How temporal aspects can be described at the system level? An ontology can be defined as a set of concepts and categories in a subject area or domain that shows their properties and the relations between the properties (Grenon and Smith, 2004; Mozersky, 2015). A temporal ontology provides a vocabulary for expressing facts about ordering relations among instants and intervals, together with information about durations, and about temporal positions, including date-time information. In CCeCE settings, time ontologies can be applied, for example, for scheduling cross-cultural meetings across time zones, scheduling joint recurrent actions between team members and monitoring the progress of cross-cultural meetings and discussions. The formal part of this study is based on the W3C OWL-Time ontology (Hobbs and Pan, 2004; W3C, 2017) and on the BFO (Grenon and Smith, 2004). The BFO, developed at the Institute for Formal Ontology and Medical Information Science (IFOMIS) at the University of Leipzig, Germany, is a theory of the basic structures of reality. In the next sections, these ontologies are briefly introduced and examples of their applications in the $\mathrm{CCeCE}$ context are given. 


\section{TIME ONTOLOGIES}

\section{OWL-Time}

The basic structure of the OWL-Time ontology is based on Allen's (1983) temporal interval calculus representing qualitative temporal information. The ontology addresses the problem of reasoning about such information. OWL-Time provides the interval relations intervalBefore, intervalMeets, intervalOverlaps, intervalStarts, intervalDuring, intervalFinishes, intervalEquals and their reverse interval relations. A proper interval can be defined as an interval whose start and end are not identical. Directionality to time is given by the "before" relation of temporal entities. If temporal entity T1 is before temporal entity T2, then the end of T1 is before the beginning of T2. An interval can have one duration but several duration descriptions, such as 1 day or 24 hours. OWL-Time defines "Duration Description" with property values years, months, weeks, days, hours, minutes and seconds. OWLTime vocabulary consists of classes, properties, datatypes and individuals, which are described in detail in W3C (2017).

As an example of applying OWL-Time, the scheduling of a cross-cultural research project web meeting across time zones is studied. Suppose that the project manager has a videoconference scheduled for 6:00 pm (Japan time, UTC+9) on November 14, 2018. A videoconference is a conference in which participants in different locations are able to communicate with each other in sound and vision. A researcher would like to have a web meeting with the project manager at 2:00 pm (Finnish time, UTC+2) on the same day, and expects the web meeting to last 45 minutes. Will there be an overlap between the teleconference and the proposed web meeting? In this case, the facts about the teleconference and the web meeting can be specified by using OWL-Time that allows a temporal reasoner to determine whether there is a conflict.

\section{An Extension of OWL-Time: Temporal Aggregates}

OWL-Time was extended by Hobbs and Pan (2004) to cover temporal aggregates. The formal expressions of temporal aggregates are defined in detail in Hobbs and Pan (2004). Temporal aggregates are collections of temporal entities. Examples of temporal aggregates are "every 3rd Wednesday in 2019" and "3 consecutive Tuesdays." Temporal aggregates are very common in collaborative research project functions, such as managing, organizing recurrent meetings, monitoring progress and scheduling teamwork and related deliverables.

The same temporal aggregate can be broken up into a set of intervals in many different ways. For example, a week can be viewed as a week or as 7 individual successive days. A minimum temporal sequence is one whose intervals are the maximum (a week), so that the number of intervals is minimal. To formalize temporal aggregates, the temporal sequence, temporal sequence member and temporal aggregate descriptions are defined.

The temporal aggregate ontology can be used to represent complex multiple-layered collections of temporal entities for scheduling collaborative research activities such as "every other week on Tuesday, Wednesday and Friday until September 14, 2019, starting on Thursday, January 24, 2019" or "every other Monday every 5th month". In practice, an extended temporal reasoner could be a component of the CCeCE system for determining appropriate recurrent meeting times among the project team members.

\section{The Basic Formal Ontology}

Upper level ontologies are used to facilitate the semantic integration of domain ontologies and guide the development of new ontologies. For this purpose, they contain general categories that are applicable across multiple domains. The difference in terminology used between separate formal upper-level ontologies can be substantial. However, the foremost dichotomy to which most formal upper-level ontologies apply is related to time and is that between "endurants" and "perdurants" (Grenon and Smith, 2004). Endurants (to endure, also known as continuants) are wholly present; that is, all their 
proper parts are present at any time they are present. Endurants are entities that can be observed as a complete concept, at no matter which given snapshot of time. Examples are material objects, such as a rock, and abstract objects, such as an organization. Perdurants, however, are entities that are only partially present, in the sense that some of their proper temporal parts (e.g., their previous or future phases) may not be present. Perdurants are entities for which only a part exists if a person looks at them at any given snapshot in time. When time is frozen, he/she can only see part of the perdurant. Perdurants are often know as processes, for example, "negotiating." If time is frozen, then only a part of the negotiation is observed. Without previous knowledge, it would be difficult to determine if the actual process is a negotiating process at all.

BFO is a theory of the basic structures of reality (Grenon and Smith, 2004). BFO has two components: a Snap ontology and a Span ontology. Snap ontology is related to endurants, and Span is related to perdurants. Span is an ontology of occurrences and more generally, an ontology of entities that have temporal parts. Span entities are divided mainly into (a) processual entities which are happenings or occurring entities, changes of various kinds in substantial entities, (b) spatio-temporal regions, that is, four dimensional regions of space-time and (c) temporal regions, that is, the whole of time and all of its parts. In this study, the focus is on Span temporal regions. The temporal regions of Span are summarized in Table 4.

\section{A Proof-of-Concept Example of Span Ontology Related to Cross-Cultural e-Collaboration}

In this section, the authors describe temporal entities related to cross-cultural project meetings and discussions between Finnish and Japanese research teams. The aim is to identify temporal regions according to the Span ontology. Temporalities of cross-cultural meetings and discussions have an effect

Table 4. Temporal regions in Span ontology

\begin{tabular}{|c|c|}
\hline Concept & Definitions \\
\hline Time $\mathrm{T}$ & The constant time designates an individual which is the whole of time. \\
\hline TimeRegion TR(x) & $\begin{array}{l}\mathrm{TR}(\mathrm{x}) \text { means that } \mathrm{x} \text { is a region of time, i.e., a part of time that may be extended or } \\
\text { instantaneous, connected to various degrees or scattered. TimeRegion }(\mathrm{x}) \equiv \operatorname{def} \operatorname{Part}(\mathrm{x}, \text { time })\end{array}$ \\
\hline TimeInstant TI(x) & $\begin{array}{l}\text { TI }(x) \text { means that } x \text { is an instant of time. TimeInstant is a specialization of the predicate } \\
\text { TimeRegion. }\end{array}$ \\
\hline TemporalLocation TL(x, $\mathrm{t})$ & $\begin{array}{l}\mathrm{TL}(\mathrm{x}, \mathrm{t}) \text { is a primitive relation between an entity and a region of time 'TemporalLocation( } \mathrm{a}, \mathrm{t}) \text { ' } \\
\text { stands for: ' } \mathrm{a} \text { is located at region of time t'. }\end{array}$ \\
\hline $\begin{array}{l}\text { TemporalLocation at an Instant } \\
\text { TLI }(x, t)\end{array}$ & $\mathrm{TLI}(\mathrm{x}, \mathrm{t})$ means that $\mathrm{x}$ is temporally located at $\mathrm{t}$ and that $\mathrm{t}$ is an instant of time. \\
\hline TemporalCo-Location TCoL(x, y) & $\mathrm{TCoL}(\mathrm{x}, \mathrm{y})$ means that $\mathrm{x}$ and $\mathrm{y}$ are located at the same temporal region. \\
\hline Temporal Subsumption TSbL(x, y) & $\begin{array}{l}\mathrm{TSbL}(\mathrm{x}, \mathrm{y}) \text { means that } \mathrm{x} \text { temporally subsumes } \mathrm{y} \text {, i.e., the temporal location of } \mathrm{y} \text { is a part of the } \\
\text { temporal location of } \mathrm{x} \text {. }\end{array}$ \\
\hline TemporalPart TP(x, y) & $\begin{array}{l}\text { Each temporal part is the sum of all co-temporal parts of a Span entity located within a given } \\
\text { region of time. } \\
\text { TemporalPart(x, y) } \equiv \operatorname{def} \operatorname{Part}(\mathrm{x}, \mathrm{y}) \wedge \forall \mathrm{z}((\operatorname{Part}(\mathrm{z}, \mathrm{y}) \wedge \operatorname{Cotemporal}(\mathrm{x}, \mathrm{z})) \\
\rightarrow \operatorname{Part}(\mathrm{z}, \mathrm{x}))\end{array}$ \\
\hline TemporalSlice TS(x, y) & $\begin{array}{l}\text { TS }(x, y) \text { means that } x \text { is an instantaneous temporal part of } y . \\
\text { TemporalSlice }(x, y) \equiv \operatorname{def} \operatorname{TemporalPart}(x, y) \wedge \exists \operatorname{AtTime}(x, t) \\
\operatorname{AtTime}(x, y) \equiv \operatorname{def}(\operatorname{TemporalLocation}(x, y) \wedge \operatorname{TimeInstant}(y))\end{array}$ \\
\hline Event & $\begin{array}{l}\text { The predicate Event is a holding of instantaneous processuals. } \\
\text { Event }(x) \equiv \operatorname{def} \exists y(\operatorname{Processual}(\mathrm{y}) \wedge \operatorname{TemporalSlice}(\mathrm{x}, \mathrm{y}))\end{array}$ \\
\hline OccursAt & $\begin{array}{l}\text { The relation OccursAt is the relation that holds between a processual entity and an instant of } \\
\text { time at which a temporal slice of this processual is located. } \\
\operatorname{OccursAt}(x, t) \equiv \operatorname{def} \exists y(\operatorname{TemporalSlice}(y, x) \wedge \operatorname{AtTime}(y, t))\end{array}$ \\
\hline
\end{tabular}


on the overall dynamics of the whole collaboration process starting from the very first contact and ending at possible collaborative actions in practice. A self-evaluation among the Finnish (University of Jyväskylä) and Japanese (Keio University) research teams was carried out by means of semi-structured theme interviews and group working. The self-evaluation was conducted from 2010 to 2017 when the collaboration was most active (Heimbürger, 2016, 2017; Heimbürger et al., 2010a, 2010b, 2012; Heimbürger \& Kiyoki, 2014; Kiyoki et al., 2016). The theme interviews had three main themes: life before, during and after a discussion process and related meetings. The interview and group working data were analyzed. The authors identified fourteen general-level sets that have temporal elements. The temporal sets and their elements can be described as follows. A logical symbol $\supset$ is used to indicate that elements in parentheses belong to a general-level set.

Set 1 - Working time $\supset\{$ Holidays, Festivals, Business Hours, Academic Terms\}: Major holidays, festivals, business hours and academic terms differ between Japan and Finland to some extent. The differences should be taken into account when scheduling collaborative actions, virtual and/ or face-to-face meetings.

Set 2 - Beginning of the Meeting: Meetings, whether face-to-face meetings or virtual, are not begun in the same way as a person moves from culture to culture. In Japan, the beginning phase takes 15-20 minutes, including a formal introduction, protocol seating, green tea, small talk and then a signal from the senior Japanese team member to begin. In Finland, the beginning phase takes 5 minutes, including making formal introductions, getting cups of coffee, sitting down and beginning.

Set 3 - Structuring a Meeting $\supset$ \{Linear-Active, Reactive\}: Linear-active members need relatively little preamble or small talk before getting down to business. They like to introduce bullet points that can serve as an agenda. Tasks are segmented, discussed and dealt with one after one other, linearly in time. The solutions reached are summarized in the meeting minutes. Finns are linear-active during meetings. Reactive individuals do not have a linear obsession with the agenda and are not wooed by multi-active arguments. In Japan, for instance, things are not black or white, possible or impossible, right or wrong. Japanese see arguments and ideas converging and ultimately, merging in time. The Japanese approach concentrates on harmonizing general principles before examining any details.

Set 4 - Meeting Behavior $\supset$ \{ Silence, Listening\}: The deliberate use of silence can be an invaluable advantage in discussions. In Finland and Japan, silence is not uncomfortable but is an integral part of social interaction. In both countries, what is not said is regarded as important, and silence can last several minutes. Listening habits can also play an important part in the negotiating process. Finns and Japanese have a good ability to listen closely for long periods of time.

Set 5 - Body Language $\supset\{$ Instantaneous Signs, Sequential Signs\}: Finns and Japanese use body language that is well understood by fellow nationals. Their body language is very subtle. Finns and Japanese are accustomed to looking for minimal signs. The signs can be instant, occasional or sequential with varying temporal intervals constituting a temporal entity.

Set 6 - Objectives: For Japanese, the current project or proposal is a trivial item in comparison with the momentous decision they have to make about whether to enter into a lasting collaborative relationship with foreigners. Japanese seek long-term relationships. Usually, decisions have been made by consensus before the meeting. Japanese see meetings as an occasion for presenting decisions, not changing them. Finns appreciate long-term relationships and can make decisions during meetings.

Set 7 - Professionalism: As far as professionalism is concerned, what is often forgotten is that negotiating teams rarely consist of professional or trained negotiators. Researchers used to directing their own nationals may lack foreign experience. When confronted with a different mindset, they are not equipped to figure out the logic, intent and ethical stance of the other side and may waste time talking past each other. 
Set 8 - Social Setting: Japanese regard a discussion as a social ceremony to which important considerations of venue, participants, hospitality and protocol, timescale, courtesy of discussion and the ultimate significance of the session are attached. Japanese view the session as an occasion to ratify ceremonially decisions that have previously been reached by consensus. Finns have little feel for the social nuances displayed by Japanese. In straightforward egalitarian cultures, meetings are conducted without regard to social status. Although quite polite, Finns have difficulty settling down to a role in meetings where social competence dominates technical know-how.

Set 9 - Values: Finns rely on data and try to get as much action and decision making as possible into the hours available. Finns have a time/efficiency equation in their mind. Japanese have their own aesthetic norms, which are bound up with a complex set of obligations. Japanese see themselves as farsighted negotiators and courteous conversationalists.

Set 10 - Compromise: It is not uncommon for discussion to enter a difficult stage where the teams get bogged down or even find themselves in a deadlock. For Japanese, compromising during a discussion is a departure from the organizational-backed consensus. Adjournment is sometimes the only way out. Compromise may be defined as finding a middle course. Japanese like to use go-betweens. Finns might rank directness above subtle diplomacy in discussions.

Set 11 - Logic: In Japan, different views co-exist in the same space and time, without invoking conflict or an urge to resolve differences. In Western logic, contradictory views of the same thing are not tolerated. Differences must be resolved. Singular and exclusive truths are looked for.

Set 12 - Decision making $\supset$ \{Before the Meeting, During the Meeting, After the Meeting\}: Discussions lead to decisions. How long they take to be made depends on the cultural groups involved. Japanese prefer to let decisions be made for them by gradually building up a weighty consensus instead of making decisions. A decision may take months. Once Japanese have made decisions, they expect their partners to move fast toward implementation. What Finns fail to understand is that Japanese, during the long decision-making process, are simultaneously making preparations for the implementation of the project.

Set 13 - Agreements: Finns regard a written agreement as something that is final. For Japanese, the contract is merely a statement of intent. They will adhere to it as best they can but will not feel bound by it if conditions suddenly change.

Set 14 - Relationship management $\supset$ \{Before the Meeting, In the Meeting, After the Meeting\}: In Japan, before the meeting, it is good to arrange an introduction by a respected third party of high status, ideally someone known to the candidate collaborator and her or his Japanese counterpart. In Finland, it is good to begin by sending a letter, an email or a fax in English with basic information about the organization and the proposal, and follow up with a phone call. If the potential collaborator is interested, a date and time for a meeting can be confirmed. During the meeting, Japanese and Finns give little gifts, and after the meeting, season's greetings are appreciated.

Temporal parts related to cross-cultural meetings and discussions and examples of temporal regions according to the Span ontology are illustrated in Figure 9. The overall concept of a perdurant entity, in this case in the form of temporal regions of the Span ontology, provides an interesting framework for analyzing temporal entities related to cross-cultural meetings and discussions, as well as for modeling the progress of the discussion and meeting processes. Meetings and discussions are social situations with a protocol that includes certain rules and forms. These rules and forms include time-sensitive parts. In cross-cultural environments, several protocols occur. These protocols can collide or harmonize depending on the participants' cultural competence. This concerns the timesensitive components of the protocols. For example, Finnish participants who are not aware of the Japanese beginning phase of the meeting that takes around 15-20 minutes would try to start the meeting at once according to the Finnish protocol. In this case, Finnish participants would cause negative tensions at the very beginning of the meeting. 
Figure 9. Temporal parts related to cross-cultural meetings and discussions in e-collaboration

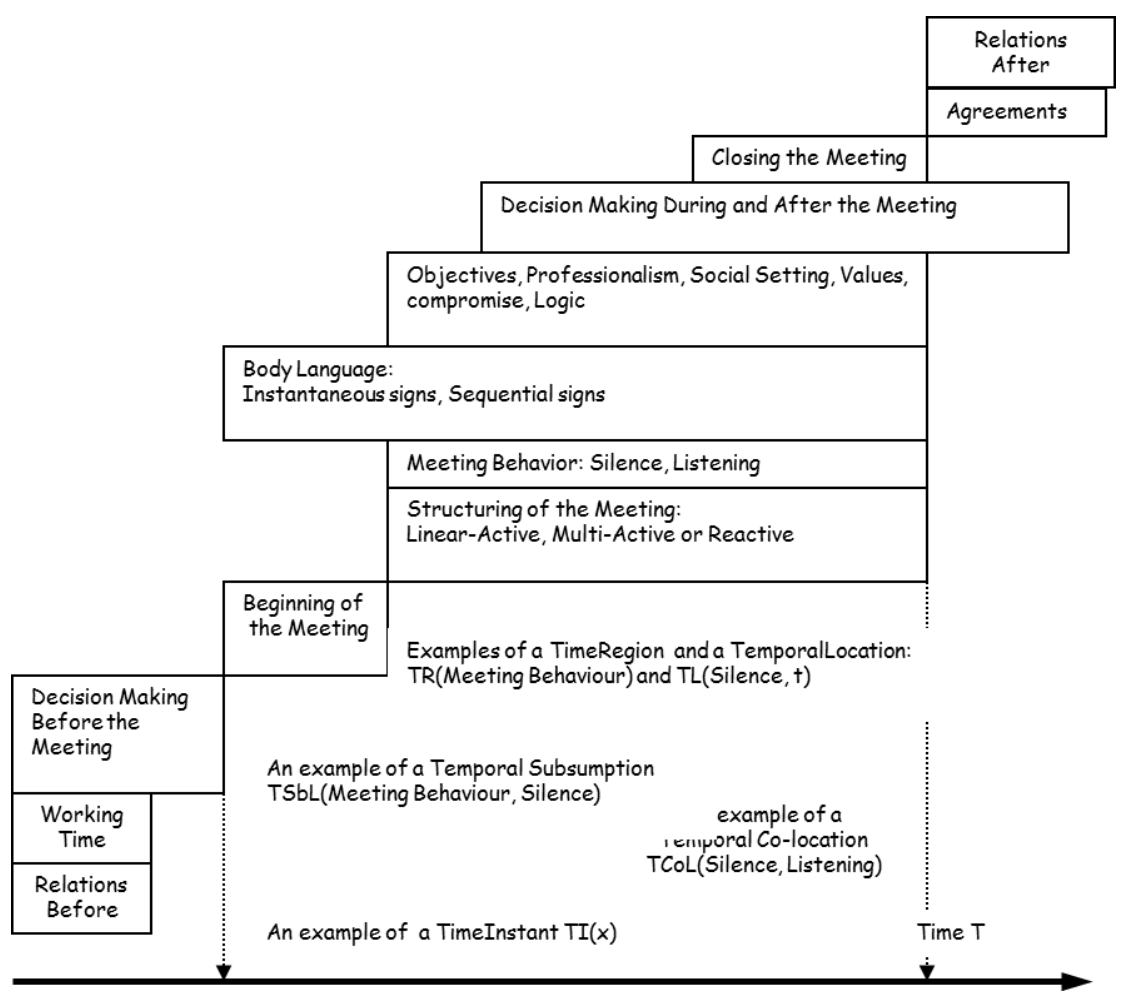

\section{CONCLUSION}

In this cross-cultural e-collaboration study, three main issues were addressed. First, cultural dimensions and time models were discussed. Second, temporal aspects related to e-collaboration activities were introduced. Third, formal ontological approaches for identifying and describing temporal entities in the context of cross-cultural meetings and negotiations were presented. The authors also provided examples of applying those ontological approaches to schedule meetings and recurrent actions across time zones and to describe temporal regions of cross-cultural meetings and negotiations in e-collaboration. Based on self-evaluation, the authors analyzed collaborative meeting and negotiation processes and identified fourteen main sets that are time-sensitive. The focus of the study has been on Finnish and Japanese cultures. The generalization of the study results is not straightforward.

The added value of this study can be summarized as follows. Identification of temporal entities of cross-cultural meetings and discussions deepen the understanding of how these social situations proceed. The ontology provides a formal approach for describing temporal entities. Formal descriptions are the basis of designing and demonstrating different components of the CCeCE system. In practice, the graphical presentation (Figure 9) of the temporal parts related to cross-cultural meetings and discussions could be the basis of a graphical interface for one of the educational components of the $\mathrm{CCeCE}$ system. The component would illustrate the temporal progress of cross-cultural meetings and discussions for project managers and team members. In addition, the results can be applied to designing a cross-cultural software agent or artificial intelligence based expert system, which will assist and support team members' work in CCeCE environment. Furthermore, the results can be applied to cross-cultural Pepper robot design (Wikipedia, n.d.a). 
Temporal aspects in cross-cultural e-Collaboration have an important role in the design and implementation of a CCeCE. This study has deepened the knowledge and understanding of temporal aspects, both informal and formal, in the $\mathrm{CCeCE}$ context. By understanding different temporal dimensions and dynamics in cross-cultural research processes, team staffs can improve their skills in cross-cultural communication and increase their cultural competence. Cross-cultural projects and teams that use CCeCE-like systems could benefit from mutual learning experiences and innovative thinking to enhance the competitive position of their organizations. From the operational point of view, cultural competence integrates and transforms knowledge about cultures, groups of people and individuals into specific practices and attitudes. 


\section{REFERENCES}

W3C. (2017). Time ontology in OWL, W3C recommendation. Retrieved from http://www.w3.org/TR/owl-time Adam, B. (2006). Time. Theory, Culture \& Society, 23(2-3), 119-138. doi:10.1177/0263276406063779

Allen, J. (1983). Maintaining knowledge about temporal intervals. Communications of the ACM, 26(11), 832-843. doi: $10.1145 / 182.358434$

Alonso, O., Gertz, M., \& Baeza-Yates, R. (2007). On the value of temporal information in information retrieval. ACM SIGIR Forum, 41(2), 35-41.

Ancona, D. G., Okhuysen, G. A., \& Perlow, L. A. (2001). Taking time to integrate temporal research. Academy of Management Review, 26(4), 512-529. doi:10.5465/amr.2001.5393887

Berry, J. W., Poortinga, Y. H., Breugelmans, S. M., Chasiotis, A., \& Sam, D. L. (2011). Cross-Cultural Psychology. Cambridge: Cambridge University Press. doi:10.1017/CBO9780511974274

Bettoni, M., Bernhard, W., Bittel, N., \& Mirata, V. (2015). eSF: An e-collaboration system for knowledge workers. In A. L. Kok, H. Lee, \& A. Kok A (Eds.), Cultural, Behavioral, and Social Considerations in Electronic Collaboration (pp. 157-172). Hershey, PA: IGI Global.

Bosch-Sijtsema, P. M., \& Henriksson, L. (2014). Managing projects with distributed and embedded knowledge through interactions. International Journal of Project Management, 32(8), 1432-1444. doi:10.1016/j. ijproman.2014.02.005

Chen, C., Chen, W., \& Ling, S. F. (2003). Project scheduling for collaborative product development using DSM. International Journal of Project Management, 21(4), 291-299. doi:10.1016/S0263-7863(02)00023-6

De Mente, B. L. (2004). Japan's Cultural Code Words. Singapore: Tuttle Publishing.

Desouza, K., \& Evaristo, J. (2004). Managing knowledge in distributed projects. Communications of the ACM, 47(4), 87-91. doi:10.1145/975817.975823

Evaristo, R., \& van Fenema, P. C. (1999). A typology of project management: Emergence and evolution of new forms. International Journal of Project Management, 17(5), 275-281. doi:10.1016/S0263-7863(98)00041-6

Ghauri, P. N., \& Usunier, J. C. (2003). International Business Negotiations. Amsterdam: Elsevier.

Grenon, P., \& Smith, B. (2004). SNAP and SPAN: Towards dynamic spatial ontology. Spatial Cognition and Computation, 4(1), 69-104. doi:10.1207/s15427633scc0401_5

Hall, E. T. (1990). The Silent Language. New York: Anchor Books.

Heimbürger, A. (2009). Temporal entities in the context of cross-cultural meetings and negotiations. In Y. Kiyoki, T. Tokuda, \& H. Jaakkola (Eds.), Information Modelling and Knowledge Bases XX (pp. 290-308). Amsterdam: IOS Press.

Heimbürger, A. (2014). Time - A multidimensional concept. In B. Thalheim, H. Jaakkola, \& Y. Kiyoki (Eds.), Information Modeling and Knowledge Base XXVI (pp. 128-139). Amsterdam: IOS Press.

Heimbürger, A. (2016). Global communication with icons in environmental contexts. In T. Welzer, H. Jaakkola, B. Thalheim, Y. Kiyoki, \& N. Yoshida (Eds.), Information Modelling and Knowledge Bases XXVII (pp. 95-102). Amsterdam: IOS Press.

Heimbürger, A. (2017). Meta-level modelling of e-education ecosystem in multicultural context. In H. Jaakkola, B. Thalheim, Y. Kiyoki, \& N. Yoshida (Eds.), Information Modelling and Knowledge Bases XXVIII (pp. 182-194). Amsterdam: IOS Press.

Heimbürger, A., Jaakkola, H., Sasaki, S., Yoshida, N., \& Kiyoki, Y. (2010a). Context-based knowledge creation and sharing in cross-cultural collaborative communities. In Y. Kiyoki, T. Tokuda, \& H. Jaakkola (Eds.), Information Modelling and Knowledge Bases XXI (pp. 76-88). Amsterdam: IOS Press.

Heimbürger, A., \& Kiyoki, Y. (2014). Context and user-centered approaches: Icons in cross-cultural context. In P. Brézillon \& A. J. Gonzalez (Eds.), Context in Computing. A Cross-Disciplinary Approach for Modeling the Real World (pp. 309-325). Springer. doi:10.1007/978-1-4939-1887-4_20 
Heimbürger, A., Kiyoki, Y., Jaakkola, H., \& Suhardijanto, T. (2012). Future directions of context modelling and cross-cultural communication. In J. Henno, Y. Kiyoki, T. Tokuda, \& N. Yoshida (Eds.), Information Modelling and Knowledge Bases XXIII (pp. 399-411). Amsterdam: IOS Press.

Heimbürger, A., Sasaki, S., Yoshida, N., Venäläinen, T., Linna, P., \& Welzer, T. (2010b). Cross-cultural collaborative systems: Towards cultural computing. In Y. Kiyoki, T. Tokuda, \& H. Jaakkola (Eds.), Information Modelling and Knowledge Bases XXI (pp. 403-417). Amsterdam: IOS Press.

Hobbs, J., \& Pan, F. (2004). An ontology of time for the semantic web. ACM Transactions on Asian Language Information Processing, 3(1), 66-85. doi:10.1145/1017068.1017073

Hofstede Insights. (2019). Country comparison. Retrieved from https://www.hofstede-insights.com/country-comparison/

Hofstede, G. (2010). Cultures and Organizations. Software of the Mind. Intercultural Cooperation and Its Importance for Survival. New York: McGraw-Hill.

Johnson, N. F., \& Keane, H. (2017). Internet addiction? Temporality and life online in the networked society. Time \& Society, 26(3), 267-285. doi:10.1177/0961463X15577279

Kiyoki, Y., Chen, X., Heimbürger, A., Chawakitchareon, P., \& Sornlertlamvanich, V. (2016). Cross-cultural and environmental data analysis in data mining processes for a global resilient society. In T. Welzer, H. Jaakkola, B. Thalheim, Y. Kiyoki, \& N. Yoshida(Eds.), Information Modelling and Knowledge Bases XXVII (pp. 281-298). Amsterdam: IOS Press.

Lewis, R. D. (2010). When Cultures Collide: Leading Across Cultures. London: Nicholas Brealey Publishing.

Mozersky, M. J. (2015). Time, Language, and Ontology. Oxford: Oxford University Press. doi:10.1093/acprof :oso/9780198718161.001.0001

Parrish, P., \& Linder-VanBerschot, J. (2010). Cultural dimensions of learning: Addressing the challenges of multicultural instruction. The International Review of Research in Open and Distributed Learning, 11(2), 1-19. doi:10.19173/irrodl.v11i2.809

Wikipedia. (n.d.a). Pepper Robot. Retrieved from: https://en.wikipedia.org/wiki/Pepper_(robot)

Shaules, J. (2015). The Intercultural Mind: Connecting Culture, Cognition, and Global Living. Yarmouth. Nicholas Brealey Publishing.

Shipp, A. J., \& Fried, Y. (2014). Time and Work. London: Psychology Press.

Takeuchi, H. (2001). Towards a universal management concept of knowledge. In I. Nonaka \& D. Teece (Eds.), Managing Industrial Knowledge: Creation, Transfer and Utilization (pp. 315-329). London: SAGE Publications. doi:10.4135/9781446217573.n16

Tang, Y., Ye, X., \& Tang, N. (2010). Temporal Information Processing Technology and Its Applications. Berlin: Springer-Verlag. doi:10.1007/978-3-642-14959-7

Wang, F. (2009). Is culture computable? IEEE Intelligent Systems, 24(2), 2-3. doi:10.1109/MIS.2009.31

Watanabe, T. (2017). The story-presenting method: A method for constructing multiple viewpoints to understand different cultures. Integrative Psychological \& Behavioral Science, 51(3), 403-418. doi:10.1007/s12124-0179391-9 PMID:28534104

Wikipedia. (n.d.b). Time standard. Retrieved from https://en.wikipedia.org/wiki/Time_standard

Anneli Heimbürger is a Senior researcher and Senior lecturer at the University of Jyväskylä, Faculty of Information Technology. Over 20 experience in Finland-Japan collaboration. Examples of areas of interest: cross-cultural communication and ICT, new pedagogical methods in e-education.

Yasushi Kiyoki is a Professor at the Keio University Shonan Fujisawa Campus (SFC) in the Faculty of Environment and Information Studies. Over 20 years' experience in Japan-Finland collaboration. Examples of areas of interest: cross-cultural computing, semantic computing, data mining. 